\title{
Quand le silence se fait : bribes de paroles de femmes sur la sexualité au XVII ${ }^{\mathrm{e}}$ siècle
}

When Silence Reigns. Traces of Women's Voices on Sexuality in the XVII ${ }^{\text {th }}$ century

\section{Sylvie Steinberg}

\section{(2) OpenEdition} Journals

\section{Édition électronique}

URL : http://journals.openedition.org/clio/9594

DOI : 10.4000/clio.9594

ISSN : 1777-5299

\section{Éditeur}

Belin

Édition imprimée

Date de publication : 1 mai 2010

Pagination : 79-110

ISSN : 1252-7017

Référence électronique

Sylvie Steinberg, "Quand le silence se fait : bribes de paroles de femmes sur la sexualité au XVII siècle ", Clio. Femmes, Genre, Histoire [En ligne], 31 | 2010, mis en ligne le 28 mai 2010, consulté le 03 mai 2019. URL : http://journals.openedition.org/clio/9594; DOI : 10.4000/clio.9594 


\title{
Quand le silence se fait : bribes de paroles de femmes sur la sexualité au XVII ${ }^{\mathrm{e}}$ siècle
}

\author{
Sylvie STEINBERG
}

Il n'y a pas à faire de partage binaire entre ce qu'on dit et ce qu'on ne dit pas ; il faudrait essayer de déterminer les différentes manières de ne pas les dire, comment se distribuent ceux qui peuvent et ceux qui ne peuvent pas en parler, quel type de discours est autorisé ou quelle forme de discrétion est requise pour les uns et les autres. Il n'y a pas un, mais des silences, et ils font partie intégrante des stratégies qui sous-tendent et traversent les discours.

Michel Foucault, Histoire de la sexualité, tome 1,

La Volonté de savoir, Paris, Gallimard, 1976, p. 38-39.

Qui cherche à capter, par-delà les siècles écoulés, les échos d'une parole féminine sur la sexualité au Grand siècle se heurte d'abord à un silence profond. Hormis quelques notations puisées dans les lettres de Madame de Sévigné ${ }^{1}$ ou de la princesse palatine, peu de traces de voix féminines sur la sexualité semblent avoir été conservées. En une époque où la moralisation des relations sexuelles est considérée comme un élément essentiel d'une piété catholique profondément réformée par le concile de Trente, les procédures de mise en silence semblent tellement rigoureuses que même les prescriptions professées par les hommes d'Église, les juristes et les médecins usent d'euphémismes, de périphrases, et procèdent à la latinisation quasi systématique des termes jugés scabreux. Quant à formuler une parole sur des pratiques sexuelles personnelles, peu d'hommes s'y aventurent et, en tout état de cause, peu utilisent l'écrit pour le faire, enfouissant

1 Voir Duchêne 2004. 
pour l'éternité interrogations et ressenti dans le secret du confessionnal ou du tête-à-tête amical. Quant aux femmes...

À bien chercher pourtant, on finit par trouver des traces balbutiantes, souvent mutilées et surtout disparates. Un premier échantillon de témoignages peut être extrait de biographies religieuses $^{2}$ écrites au XVII ${ }^{\mathrm{e}}$ siècle sur des femmes dévotes jugées dignes de mémoire. Ils dépeignent le destin de femmes qui s'étant vouées à Dieu dans les couvents et monastères de la Contre Réforme ou ayant joué un rôle dans le siècle en créant compagnies charitables ou institutions éducatives à destination des filles, ont ainsi participé à l'immense vague de piété féminine qui a caractérisé l'époque. "Paroles de femmes", est-ce bien le terme idoine, demandera-t-on ? La plupart des écrits biographiques furent écrits par des directeurs de conscience et autres supérieur-e-s de couvent suivant une élaboration souvent complexe qui procédait par la réécriture de mémoires autographes rédigés sur demande et de témoignages des proches. Ceux qui relatent ces pieuses vies n'ont certainement pas beaucoup d'éléments à livrer sur le sujet de la sexualité, le chapitre obligé sur les vertus de chasteté manifestées par la religieuse tenant lieu en général de tout récit. Cependant, quelques faits méritent d'être retenus : avant de répondre à l'appel de Dieu, ces femmes, pour la plupart nées entre 1590 et 1640, ont été des petites filles (presque) comme les autres, des filles à marier et parfois des épouses, ce qui oblige leurs biographes à élaborer des récits dont la tonalité "réaliste" laisse à penser qu'ils sont puisés dans des anecdotes vécues. Certains de ces écrits se présentent sous une forme autobiographique, quoiqu'ils aient été passés au crible par leurs commanditaires et leurs éditeurs, autocensure et censure travaillant de conserve pour ôter aux textes leurs aspérités ${ }^{3}$. On doit aux éditeurs récents de certains textes, en particulier ceux de Jeanne Marie Guyon, cette célèbre quiétiste persécutée sous le règne de

2 Pour une présentation de ce corpus, composé de centaines de titres imprimés et manuscrits, ainsi qu'une étude des pratiques corporelles des religieuses, voir Le Brun 1986 : 389-408. Pour une étude de la sainteté, Albert 1997. On trouvera en fin d'article la liste des sources retenues, parmi d'autres non mentionnées qui se sont révélées infructueuses.

3 De ce fait, la limite entre les deux genres biographiques et autobiographiques est indécise. Voir les remarques de Nicole Pellegrin 2003 : 57. 
Louis XIV, ou de Marcelline Pauper, plus obscure fondatrice d'une congrégation du Nivernais, d'avoir rétabli les passages censurés par leurs éditeurs ${ }^{4}$. Sans surprise, l'ensemble de ces textes nous offrent une vision inquiète de la sexualité, marquée par l'horreur du "péché de chair", la crainte de la "tentation" et une aspiration inextinguible à la chasteté.

Autres réceptacles d'une parole féminine, les sources judiciaires léguées par les tribunaux de l'Ancien Régime ne sont pas celles qui nous offrent l'image la moins «tragique $»^{5}$ de la sexualité puisqu'elles émanent de femmes qui viennent devant les tribunaux d'Église (Officialités) pour demander un arbitrage pour des promesses de mariage non tenues ou pour faire annuler leur mariage du fait de l'impuissance de leur maríb. Doit-on, là encore, qualifier de "paroles" un ensemble de bribes filtrées plusieurs fois par des conseils (parents, amis et avocats éventuels), puis par des médecins experts et enfin par des juges enquêteurs et des greffiers? Les uns suggèrent des voies de procédure, les autres fournissent des explications physiologiques et anatomiques, les derniers lissent la parole des plaignants de manière à la faire entendre sans blesser la pudeur des juges d'Église. La longueur des procès - quelques mois à plusieurs années - donne d'ailleurs beaucoup d'occasions aux parties de parfaire leur parole en

4 La Vie de Jeanne Marie Guyon, écrite par elle à la demande de son directeur le père La Combe, a d'abord été refusée et détruite en 1681 puis, dans une nouvelle version, éditée par Pierre Poiret qui l'a expurgée en 1720. L'édition de Dominique Tronc restitue les passages supprimés par Poiret (Jeanne Marie Guyon, La Vie par ellemême et autres écrits biographiques, édition critique avec introduction et notes par Dominique Tronc, Paris, Honoré Champion, 2001) ; l'édition moderne de la vie de Marcelline Pauper (L'Expérience mystique de Marcelline Pauper, religieuse de la congrégation des sceurs de la Charité et de l'instruction chrétienne de Nevers. Étude critique de ses écrits. Avec le concours du père André Ravier s j., Nevers, Couvent saint Gildard, 1982) restitue des passages qui avaient été censurés par le P. Marcel Bouix dans son édition de 1871 (P. Marcel Bouix, Vie de Marcelline Pauper de la congrégation des scurs de la Charité de Nevers écrite par elle-même, Nevers, Fay, 1871).

5 Jean-Louis Flandrin avait noté que les amours que nous connaissons le mieux sont précisément les amours tragiques qu'ont à traiter les tribunaux, ce qui est un biais important de la recherche (Flandrin 1975 : 200-235.)

6 Sur la procédure en usage devant les Officialités, voir Darmon 1979. Sur la définition canonique de l'impuissance, d'Avack 1949. 
un discours convaincant. Sans que s'y mêlent nécessairement le calcul, le mensonge ou la tartufferie, il s'agit de donner une version crédible des faits en fonction de la définition préétablie par l'Officialité de ce type de cas judiciaire. Parmi les procès, ceux qui visaient l'annulation de mariage pour cause d'impuissance retenus $\mathrm{ici}^{7}$, requéraient davantage de descriptions circonstanciées que ceux pour promesses de mariage non tenues où le témoignage de la fille séduite était souvent réduit à quelques tournures stéréotypées - l'homme avait « eu sa compagnie charnelle», l'avait «abusée », avait " joui d'elle», avait pris «sa copule charnelle $»^{8}$. L'interrogatoire était mené de façon serrée en reprenant terme à terme les déclarations de l'autre époux pour éclaircir la nature des relations conjugales, ce qui avait pour effet d'obliger les deux parties à adopter une tactique de défense en apportant des éléments contradictoires, donc à préciser leur propre vision des faits, à livrer leur propre parole.

Toutes ces manipulations, ces manœuvres, ces euphémisations, ces mensonges mêmes, ne sont des obstacles que si l'on cherche à découvrir un secret derrière les anecdotes, les souvenirs, les plaintes judiciaires ou les interrogatoires. Chaque parole de femmes est d'abord un arrachement au silence et nulle d'entre elles n'a eu véritablement le désir de confier des détails sur sa vie sexuelle. Plus que des secrets, il y derrière chacune de ces paroles des silences et, paradoxalement, il y a plus de probabilité d'atteindre aux mécanismes de ce silence qu'à la sexualité "réelle". Depuis les fantasmes et les tourments liés au sentiment de péché dans la toute petite enfance en passant par les premières expériences infantiles et adolescentes jusqu'aux premiers temps des relations conjugales, le silence fut sans doute un puissant instrument de contrôle de la sexualité féminine en même temps que le principal élément du vécu de cette sexualité par les femmes qui en livrèrent quelques bribes.

7 La présente étude s'appuie sur une enquête dans les fonds des Officialités parisiennes (années 1615-1618 et 1622-1625 pour l'Officialité de Paris ; épaves de l'Officialité de Saint-Germain-des-Prés, années 1655-1665).

8 Pour la procédure qui a cours pour les promesses de mariage non tenues, voir Demars-Sion 1986. Relevé du vocabulaire désignant les relations sexuelles par Millinaire $2006: 125-127$. 


\section{Simplicité, pureté, innocence : rêves et fantasmes de petites filles}

Les fillettes que dépeignent les biographies spirituelles sont vouées à deux types de destinée : soit elles ignorent tout du péché, plaçant d'emblée leur corps sans souillure sous l'égide de celui de la mère de Dieu, soit, nouvelles Madeleine - dont la figure connaît un regain de dévotion au XVII ${ }^{\text {e }}$ siècle -, elles avouent avoir fait l'expérience de la "tentation". Quoique les deux scénarios puissent être aisément rattachés à une branche de la tradition hagiographique, aucun des deux n'aurait pu avoir de vraisemblance pour leurs lecteurs contemporains si le silence des adultes qui élevaient ces fillettes n'avait ouvert la voie soit à la totale ignorance soit à la détresse devant l'impossibilité de faire part de son "péché".

Le premier scénario, celui de la fillette innocente dont le destin est quasi scellé dans le ventre de sa mère, se trouve principalement développé par les ecclésiastiques, hommes ou femmes, qui rédigent des biographies religieuses plutôt que dans les mémoires autographes. N'eût-il pas été en effet présomptueux qu'une femme pieuse, fût-elle reconnue sainte de son vivant, affirmât elle-même qu'elle avait toujours tout ignoré du "péché" ? Encore quelques-unes de celles qui écrivirent leurs mémoires signalèrent-elles cependant leur désir enfantin de pureté et insistèrent-elles sur leur prédisposition à la chasteté. Pour les biographes, évoquer le destin d'une fille absolument exempte de toute connaissance de la sexualité répondait évidemment à plusieurs desseins propres à ce genre d'exercice: éduquer, exalter, donner pour exemple, témoigner. Malgré (ou précisément grâce à) ce caractère normatif, le premier scénario permet de mesurer à quel point l'ignorance revêtait un caractère sacré aux yeux de l'Église. Ainsi, l'auteur anonyme de la Vie de Marguerite du Saint Sacrement, femme réputée pour avoir gardé la pureté de son baptême durant toute son existence, établit de subtiles distinctions entre la simplicité qui, dit-il, "consiste en une droiture d'esprit et de cœur, éloignée de toute vaine prudence, de toute tromperie, de tout artifice, accompagnée d'une naïveté, et d'une franchise dans les paroles et dans les actions », la pureté qui, ajoute-t-il, "est une exception de tout mélange de ce qui est soüillé et prophane, avec ce qui procède de la grâce » et, enfin, l'innocence qui est «non 
seulement un éloignement du péché, mais encore un état saint qui est source de toute vertu, et qui est proprement une naissance en Dieu, telle qu'elle serait si le baptême avoit déraciné toute notre concupiscence et que l'amour propre se fust converty au seul amour de Dieu. » Néanmoins, l'auteur reconnaît au passage que la différence entre ces divers états et l'ignorance n'est pas toujours perceptible aux yeux du commun. Et il avoue: la simplicité, "parce qu'elle est contraire à la finesse générale des hommes, [...] passe bien souvent pour ignorance, quoy que toutefois elle soit d'autant plus remplie de sagesse, qu'elle est éloignée de la fraude et du mensonge, et qu'elle marche droit dans la voye de la vérité $»^{9}$. Innocence ou ignorance, quelle petite fille aurait été véritablement capable de faire le départ entre les deux notions? Quel parent dévot n'eût pas souhaité qu'on qualifiât de sainte innocence la naïveté de son enfant?

À côté des explications théologiques, les anecdotes qui exaltent l'innocence des fillettes ont souvent une facture réaliste qui laisse à penser qu'elles sont bâties à partir de mémoires préexistants, de conversations et de témoignages. Le soupçon qu'elles sont en partie reconstituées n'empêche pas d'y lire la manière dont la simplicité des petites dévotes se nourrit d'abord du lourd silence qui entoure les réalités du sexe et s'accroche aux euphémismes qu'elles entendent prononcés à l'occasion du prêche, du catéchisme et des lectures pieuses dont elles sont manifestement friandes. Le référent de tous ces mots qui disent la chute, la chair, la virginité ou encore la pureté leur échappe et elles les interprètent à partir de leurs expériences et préoccupations de petites filles. Un jour, Agnès Galland, future Agnès de Jésus, fille d'un pauvre coutelier du Puy, provoque ainsi le rire de ses voisins et le courroux de sa mère :

Il n'y avoit qu'à prononcer en sa présence les mots de virginité et de Vierge, pour mettre son cœur dans la joye. Comme chacun remarquoit en elle cette disposition, cela causa un jour un événement assez récréatif, qu'il ne sera pas hors de propos de rapporter icy. Estant allée à la boucherie ce jour-là par l'ordre de sa mère, une bouchère leur présenta un agneau qu'on venoit de tirer du ventre de sa mère tout fraischement tuée, et luy dit «Tenez Agnez, voilà de la chair vierge ». Elle agréant beaucoup cette parole donna sans marchander tout ce qu'on voulust de

$9 \quad$ La Vie de Marguerite du Saint Sacrement 1655 : 533-534. 
l'agneau, le prit avec empressement, courut à sa mère pour lui monstrer sa belle amplette, et luy dit toute joyeuse : «Ma mère, voilà de la chair vierge ». Et voyant que sa Mère rebutoit son achapt et se fâchoit de l'argent qu'elle y avoit mis, elle se mit à loüer cet agneau pour sa virginité ; et à soûtenir que la chair vierge valoit d'avantage que d'autre viande. ${ }^{10}$

Comme personne ne cherche à lui expliquer les raisons de la moquerie dont elle est l'objet, le silence s'épaissit encore autour du mystère de l'engendrement. Encore Agnès saisit-elle les raisons du rire des adultes au moment où, devenue supérieure d'un couvent, elle « raconte cela à ses sœurs comme un trait enfantin pour les récréer ». Mais d'autres anecdotes de sa Vie s'attachent - et s'amusent aussi, car même le lectorat d'une Vie spirituelle était convié à s'amuser d'une telle simplicité - à montrer qu'à cet âge avancé, elle ne connaît toujours pas le sens du mot «tentation», considérant que les religieuses sont bien aises d'échapper aux tortures qu'a subies saint Jérôme, ou encore celui du terme "violer», dont elle s'étonne qu'il provoque l'effroi car elle le donne pour synonyme «d'attacher à la robbe d'une personne un morceau d'étoffe violette $»^{11}$. Ce qui récrée ses sœurs mais, cette fois, à son insu. L'agneau, la chair, la naissance miraculeuse du Christ, autant de symboles et de points de doctrine qui informent la vision du monde de la petite Agnès, bien loin de l'acception prosaïque qu'en donne une bouchère facétieuse.

On retrouve le même genre d'interprétation du réel chez Madeleine Vigneron, née en 1628 dans une famille d'honnêtes bourgeois de Senlis. À plusieurs reprises, à partir de l'âge de vingtcinq ans, elle se livre en écrivant des mémoires sur sa vie que son directeur de conscience reprendra pour les éditer et les commenter. Elle relate qu'un jour, le Christ lui apparut pour lui révéler qu'elle était prédisposée à la chasteté du fait des souffrances que sa mère avait endurées durant sa grossesse, «ces souffrances luy ayant été données pour la préserver du péché particulièrement durant ce temps, d'autant que si elle en eût commis quelqu'un, elle se seroit rendüe indigne de [sa] protection sur ton corps? $»^{12}$, précise-t-il. Madeleine en conçoit

10 La Vie de la Vénérable Mère Agnez de Jésus $1666: 31$.

11 Ibid. : 400-401.

12 Bourdin $1679: 7$. 
une véritable passion pour la virginité, qu'accompagne une pudeur extrême qui s'est manifestée bien plus tôt :

La moindre nudité du corps m'étoit insupportable si petite que je sois, même au berceau quand on change les petits enfans, j'en avois horreur; ma nourrice s'en appercevant me tenoit le plus caché qu'elle pouvoit, c'est à quoy j'ay toûjours eu une singulière dévotion. ${ }^{13}$

Sa petite enfance est un véritable chemin de croix sur lequel elle ne cesse de braver la maladie et les accidents. Vers l'âge de quatre ans, elle trébuche sur la margelle d'un puits et se retrouve accrochée par un pied au-dessus du vide. Cris, prières, mouvements, tout le monde accourt et elle est enfin délivrée. Mais le soulagement d'être secourue fait bientôt place au désespoir. Croyant être en présence d'anges, elle se lamente : «Ô assurément je ne croy pas être dans les bonnes grâces de mon bien aimé, le monde a vû le petit corps, je ne seray jamais vierge $»^{14}$. La promesse, un temps entrevue, de réitérer le baptême ad mortem dans les eaux sombres d'un puits de ferme est brutalement rompue par le spectacle pathétique d'un «petit corps » ridiculement pendu par les pieds, jupe par dessus tête. Ce qu'est la virginité pour la petite Madeleine Vigneron? Des efforts à sa mesure d'enfant pour rester digne de siéger parmi les anges, cachée aux yeux des autres, des efforts qui la soulagent des tourments qu'elle endure quotidiennement dans son corps, un état de pureté opposé à une souillure métaphorique, qu'elle a extrapolé de celle de son lange ou de ses effets maculés par la maladie ${ }^{15}$.

Le silence qui entoure les relations sexuelles et l'exaltation continuelle de la virginité ne manquent pas de provoquer de véritables terreurs. Paradoxalement, ce sont ces fillettes qui sont censées ne rien savoir du "péché de chair", ces petites fiancées du Christ, qui manifestent la plus grande peur de se trouver en présence de garçons. Ainsi en est-il de Galiote de Sainte Anne, née en 1588,

13 Ibid. : 14.

14 Ibid. : 36.

15 Sur le corps des religieuses, leur rapport à la souillure, au sang, à la maladie et à la mortification, voir Bynum 1994 ; Albert 1997 et la synthèse de Jacques Gélis 2005. 
tellement prédestinée à devenir pieuse qu'on relate même qu'elle fut allaitée dans un monastère et fit profession à onze ans :

On eut dit dans ses commencements qu'elle eut apporté cette vertu du ventre de sa mère, car dès son plus bas âge, auquel elle n'avoit point de connoissance et d'usage de la raison, elle abhorroit les hommes, et fuyoit leur compagnie comme la peste, même celle de ses frères, n'en voulant jamais voir, ny avoir en ses plus innocentes récréations ${ }^{16}$.

Cette fuite s'étend parfois à tous les membres mâles de la famille, aux domestiques bien sûr qui, plusieurs anecdotes le soulignent, peuvent se montrer indélicats - l'un d'eux ne jeta-t-il pas Madeleine Vigneron sur un lit? -, et sont en tout état de cause soupçonnés d'avoir la parole trop leste, voire au père de famille. Née en 1612 dans une famille relativement modeste d'Aix, Marie Magdeleine de la Trèssainte Trinité a, d'après son biographe - qui avoue cependant avoir peu d'éléments pour reconstituer son enfance - «tant d'horreur et tant d'aversion pour la compagnie et les caresses des hommes, son Père même n'ayant qu'à l'appeler, et luy témoigner vouloir la caresser, pour l'obliger à fuir et se cacher $\|^{17}$. Le biographe d'Agnès de Jésus, quant à lui, associe à un rêve enfantin la peur des hommes qu'elle manifestera toute sa vie. Par trois fois, elle rêve qu'elle est assaillie par une troupe d'hommes «terriblement laids et rouges de visage » qui veulent la faire passer par le chemin pierreux d'une montagne puis la mener dans un pré et la battre : " elle ne pouvoit en envisager aucun, et en parlant d'eux, elle les appeloit "Aqueycharviras" c'est-à-dire dans le langage du païs, "ces visages de travers" comme si elle eût vu en chacun d'eux quelque chose de monstrueux $»^{18}$. Héroïque, la défense de la virginité devient pour cette toute petite fille un combat nocturne terrifiant contre les méchants violeurs de bergères qui arpentent les monts Golgotha de l'Auvergne, le visage rouge de désir, le visage rouge des hommes réputés luxurieux et colériques, celui des diables qui ornent immanquablement les murs de l'église paroissiale.

\footnotetext{
16 Thomas d'Aquin de Saint Joseph $1633: 410$.

17 Piny $1679: 7-8$.

18 La Vie de la Vénérable Mère Agnez de Jésus 1666: 36.
} 


\section{La honte des petites pécheresses}

La difficulté à cerner véritablement ce qu'est le "péché" qu'on leur cache se retrouve d'une autre manière chez celles qui, longtemps après, avouent avoir vécu, étant enfant, des expériences sexuelles. Dans leurs familles règne un silence pesant qui ne leur permet pas de mettre de paroles sur ce qu'elles vivent. Des années après, elles peinent d'ailleurs à trouver des mots pour le dire et emploient - ce qui ne gâte en rien la saveur de leur langue- une suite d'euphémismes, de circonlocutions élégantes, d'allusions qui laissent deviner un jeu, un geste, une affection, et retracent ce temps de l'insouciance où ne s'amoncellent pas encore sur leur front le remords et la conscience de la faute commise. Là encore, le fantasme prend parfois le pas sur la réalité. Madeleine Vigneron relate la manière dont elle aurait été "corrompue" si elle n'avait cédé à son instinct de vierge :

Je pouvais avoir encore cinq ans, quand un jour une petite fille, que je croyois dans toute l'apparence extérieure être une de mes compagnes, me vint trouver dans ma chambre, où après m'avoir demandé familièrement à quoy nous joüerions, elle me proposa un certain petit jeu, disant qu'il étoit ordinaire parmi les filles de sa connoissance, je n'en sentis pourtant que de l'aversion ne le trouvant pas honnête, et témoignay beaucoup de répugnance mais voulant m'y contraindre par force, parce qu'elle étoit plus grande que moy, Dieu sçait quel fut mon trouble, moy qui dès le berceau avois eu tant d'horreur de la nudité qu'elle m'étoit insupportable, même dans les actions les plus nécessaires, élevant à l'instant mon cœur à Dieu, criant, pleurant, je dis : non; résolument je n'en feray rien, car Dieu et tout le paradis nous voit, à ces mots, je me sentis si rudement jettée contre terre, que je croyois avoir la tête cassée, et au même moment elle disparut: Je me trouvay pourtant sans aucune blessure, reconnoissant visiblement que c'étoit le démon qui avoit pris la figure de cette petite compagne ${ }^{19}$.

Dans le récit d'enfance de Madeleine Vigneron, la figure de cette petite fille corruptrice fait pendant à celle d'un petit garçon pauvre recueilli dans la rue et nourri chez elle jusqu'au moment où il disparaît et qu'elle comprend qu'elle a, durant tout ce temps, fait l'aumône au Christ lui-même. Le recours au récit de fantasmes relève-t-il du refoulement de scènes bien réelles? Il est d'abord la reprise d'un

19 Bourdin 1679:44-45. 
modèle ancien de récit, qui met en scène des pères du désert luttant contre leurs fantasmes sexuels ${ }^{20}$, topos que l'on retrouve dans les biographies féminines après l'entrée au couvent. Cette scène fait cependant également écho à des confessions similaires de la part d'autres femmes, Marcelline Pauper et Jeanne Marie Guyon en particulier, qui décrivent la manière dont elles ont été initiées à des jeux sexuels par d'autres fillettes.

D'une certaine façon, c'est aussi le silence qui a conduit à ces expériences entre fillettes. C'est du moins l'interprétation qu'en donne Madame Guyon qui note que, vers treize ou quatorze ans, elle était «si forte ignorante [du péché qu'elle] croyait que pour le commettre, il fallait faire des immodesties avec une personne de différent sexe, que tout ce qui n'était point cela était permis $»^{21}$. Ce n'est donc pas parce que la mixité est proscrite que les expériences se partagent entre filles mais bien plutôt parce que les garçons sont déjà perçus comme des dangers à éviter à toute force. Fréquemment dans les biographies religieuses, des scènes de jeux dans les rues ou les champs sont relatées, principalement, comme chez Agnès de Jésus, pour exposer les occasions où la future dévote a pu échapper aux poursuites mal intentionnées d'un garçon. De même, les occasions de jouer avec des garçonnets lors des visites chez les parents ou les amis permettent de mettre en scène une fillette en larmes fuyant un petit ami tout surpris qui veut la caresser d'une «façon qu'il croit innocente $»^{22}$. Pour les auteurs de biographies pieuses, sans doute s'agit-il d'apporter la preuve que la mixité est dangereuse et qu'il faut la bannir de l'école, des assemblées, des pèlerinages, comme s'efforcent de le prôner les autorités ecclésiastiques ${ }^{23}$. Finalement, dans ces enfances où règne la mixité, ce à quoi les parents semblent davantage veiller, c'est à empêcher leurs enfants de fréquenter les enfants des basses classes. Madame Guyon relate que sa mère, désinvolte et peu encline à s'occuper d'elle, manqua un jour à cette surveillance et que ce fut son père qui remédia à ce coupable

\footnotetext{
20 Brown $1995: 285-286$ et 503-505.

21 Guyon $2001: 148-149$.

22 Bourdin $1679: 38$.

23 Flandrin 1975 : 106-108.
} 
relâchement en décidant illico de la conduire chez les Ursulines: «Comme ma liberté augmentait chaque jour, elle fut si loin qu'un jour je sortis de la maison et allai dans la rue avec d'autres enfants jouer à des jeux qui n'avaient rien de conforme à ma naissance $»^{24}$, note-t-elle. Crainte que les jeux des enfants des milieux populaires ne soient pas si innocents que cela? Implantée surtout dans une partie de la vieille noblesse provinciale, chez les officiers du roi, parmi les membres de professions intermédiaires-médecins, marchands, boutiquiers aisés -, la piété dévote ne contribua pas peu à rejeter sur les milieux populaires, mais également sur les milieux mondains de la ville et de la cour, l'accusation d'un libertinage désordonné, dessinant un nouveau partage du bon comportement sexuel.

C'est donc finalement dans les maisons religieuses où elle fut placée que Madame Guyon a été initiée par d'autres fillettes. Le premier séjour chez les Dominicaines où elle fut mise à l'âge de dix ans pour être redressée de ses "insolences» ne la garda pas des "mauvaises fréquentations" puisqu'elle y fit la rencontre d'une jeune fille appartenant très visiblement à un milieu inférieur au sien qui y avait été recueillie "par charité ». Celle-ci, relate-t-elle, "avait de l'esprit et deux fois mon âge, j'étais presque toujours avec elle, car j'étais sans conduite et sans occupation, et ce fut mon malheur (...) Cette fille donc me fit faire un péché. Il est vrai que je ne savais ce que je faisais ni pourquoi je le faisais, étant si jeune. Je crois que l'autre le savait bien $»^{25}$. C'est encore dans un couvent, celui des Ursulines où son père l'a conduite après l'avoir cueillie dans la rue, qu'elle renoue avec ses pratiques antérieures. Elle y rencontre deux autres filles qui lui apprennent, dit-elle, des péchés qu'elle avait ignorés jusqu'alors et auxquels elle s'adonne «follement». C'est l'ignorance qui l'y pousse ainsi que la curiosité de savoir de quoi on lui parle. Lucidement, Madame Guyon tempère tout de même la gravité de ces plaisirs infantiles, passant du plan du péché à celui de la physiologie, qui lui fait considérer à la fois son immaturité physique et son « tempérament » peu luxurieux :

\footnotetext{
24 Guyon $2001: 116$.

25 Ibid. : 125.
} 
Je ne tombais pas fréquemment dans ces péchés tant à cause que mon extrême jeunesse ne me le permettait pas, que parce que mon tempérament y était opposé, car dans tout le temps que je suis tombée dans ces désordres, je n'ai jamais eu de mouvements sensuels qu'une fois, encore fût-il très léger. Je fus près de huit mois à continuer de temps en temps mes péchés sans croire d'y faire aucun mal et sans m'en confesser. Ce fut dans cette funeste ignorance que j'en contractai l'habitude qui me fut si difficile à perdre. ${ }^{26}$

Ces jeux sexuels suggérés au départ par une ou plusieurs fillettes initiatrices se prolongent ensuite dans la solitude et le secret, laissant la fillette seule avec sa "faute". Marcelline Pauper, née en 1663, enrôlée en 1687 dans une congrégation naissante vouée aux œuvres de charité, relate elle aussi qu'elle fut « corrompue » à l'âge de sept ans par des petites filles qui lui apprirent « des jeux criminels où la pudeur et la modestie n'étaient point gardés ». Placée chez les Ursulines à huit ans, elle fait vœu de chasteté un an plus tard, ce qui ne manque pas de provoquer chez elle des peines intérieures :

Je fis ma première communion à onze ans. Ces bonnes religieuses prirent grand soin de m’y préparer. Pendant ce temps-là, ma vie paraissait assez innocente à celles qui me gouvernaient ; cependant je revenais à mon particulier à ces plaisirs criminels que l'on m'avait appris. Toutes les fois que cela m'arrivait j'en étais reprise intérieurement et remplie de confusion lorsqu'il me fallait prier Dieu. ${ }^{27}$

Au milieu du silence des adultes, le tourment naît de ne pouvoir évaluer la gravité de l'acte commis, de ne pouvoir lutter seule contre la réitération du plaisir, de ne pouvoir même mettre un mot sur ce qui est commis : «immodestie», "mouvement sensuel», "plaisirs criminels», «maudite science», «malheureux jeux», « jeux criminels », autant d'euphémismes qui laissent la fillette, puis la femme adulte qui relate les faits, aux prises avec ses doutes. Cependant, le fait que ces jeux sexuels soient partagés par des enfants du même sexe semble minorer le "péché" comme le minore le fait qu'il se prolonge dans la solitude.

À qui donc s'adresser pour connaître la nature exacte de la faute commise? Les autres fillettes s'avèrent être des informatrices peu

\footnotetext{
26 Ibid. : 148-149.

27 L'Expérience mystique de Marcelline Pauper 1982 : 12.
} 
fiables. L'une d'elle, plus âgée, plonge Marcelline Pauper dans la plus grande affliction en lui apprenant qu'elle ne cesse de commettre des " péchés mortels ${ }^{28}$. Dans les cercles qu'elles fréquentent, le silence est trop épais pour que puissent filtrer des informations utiles des conversations qui réunissent amies et parentes. Au reste, sont-elles sans doute trop pudiques pour oser franchir cette barrière du silence. N'ont-elles pas dans leur entourage ce genre de petites filles exemplaires qui se comportent comme de véritables censeurs des mœurs, générant pas leur attitude même la honte et la retenue de leurs compagnes? Née en 1578, la future carmélite Madeleine de Fontaines est de celles-là :

À peine avoit-elle atteint l'âge de la raison que l'on vit paroistre en elle une honnesteté, et une pudeur, qui donnoient de l'amour et de l'admiration tout ensemble. (...) Bien loin qu'il sortist de sa bouche une seule parole trop libre, ses oreilles ne pouvoient mesme les supporter (...) Sa seule présence inspiroit dans les autres la modération et la retenuë; et nous avons déjà remarqué au commencement de sa vie, qu'une jeune demoiselle, qui avoit accoutumé de la fréquenter et qui en la compagnie des autres se laissoit souvent emporter à dire des paroles d'une gayeté, qui passoit les bornes de la modestie, réprimoit cette liberté, et mettoit un frein à sa langue aussitost qu'elle avoit la petite Demoiselle de Fontaines pour témoin de ses discours. ${ }^{29}$

Restait alors, pour se libérer de la douleur de ne savoir la gravité de son "péché", la voie de la confession dont on sait qu'elle est devenue un élément important de la pratique religieuse rénovée par le concile de Trente. Mais la confession n'est pas un acte si aisé à accomplir. Les témoignages laissés par les femmes dévotes tendraient plutôt à montrer que les petites filles qu'elles furent y répugnèrent souvent. Les fillettes ont d'abord à démêler elles-mêmes la question de savoir ce qu'il leur faut confesser. Sont-elles des pécheresses endurcies? Peut-on qualifier leurs attouchements de "péché de chair"? Alors que toute attitude corporelle non contenue ou tout «mot à deux ententes » sont considérés déjà comme faisant "couler insensiblement le péché, comme un secret poison dans l'âme $»^{30}$, dans

\footnotetext{
28 Ibid. : 44. Passage censuré dans l'édition Bouix 1871.

29 La Vie de la Mère Magdeleine de Saint Joseph 1670 : 658.

$30 \quad V i e$ de damoizelle Elizabeth Ranquet 1655 : 77-78.
} 
quel état est donc leur pauvre petite âme? Lorsque Jeanne Marie Guyon prépare sa première communion - elle a alors onze ou douze ans - elle hésite à avouer à son confesseur une faute dont elle n'arrive pas à évaluer la malignité : " un regard ou une petite liberté avec une personne qui [lui] était très proche » sont-ils d'effroyables péchés? Suit alors le récit d'une scène qui exprime bien les inconvénients que Madame Guyon devenue femme ne cessa ensuite de trouver à la confession, elle qui, de fait, préférait ouvrir son cœur à Dieu qu'à ses serviteurs $^{31}$ :

J'allai à confesse et comme la honte commençait à me venir et que le confesseur qui n'était pas dans un confessionnal mais sur un siège, me regardait fixement et qu'il me prît même avec hardiesse le menton pour me mieux regarder, j'eus tant de confusion que je n'osai jamais lui déclarer mon péché, je ne laissai pas d'aller communier de cette sorte ; je fis peut-être un petit sacrilège (...) et j'eus beaucoup de douleur du sacrilège que j’avais fait. Comme je conçus que c'était le confesseur qui en était la cause, je le quittai, j'eus même une certaine horreur de lui, j'en pris un qui me servit beaucoup car je le craignais. ${ }^{32}$

Dans un deuxième épisode, elle relate une autre forme de scrupule qui la saisit alors qu'elle a treize ou quatorze ans à propos du plaisir qu'elle prend seule et secrètement. Au lieu d'avouer au prêtre ses actions, elle feint d'en avoir seulement eu la pensée ${ }^{33}$. Marcelline Pauper, quant à elle, se fait instruire discrètement de la gravité de ses agissements mais se garde bien de les avouer.

À cause de cette complexité à identifier réellement la nature et la gravité des actes commis, ce n'est qu'à dix-huit ans que la future Marie de l'Incarnation franchit le pas de se confesser de ses péchés enfantins, dont elle ne dit d'ailleurs pas la véritable nature : «Et si j'eusse cru que mes récréations d'enfant et autres passe-temps que depuis cet âge j'avais pris avec mes compagnes eussent été péchés, je m'en fusse bien vite confessée, mais ne le croyant pas, je ne le faisais pas ». La même déception la saisit lorsqu'elle se retrouve face au prêtre censé lui apporter le réconfort :

\footnotetext{
31 Sur Madame Guyon et la confession, voir Gondal 1989 : 99.

32 Guyon 2001 : 134-135, passage censuré.

33 Ibid. : 149-150.
} 
Alors je me disposai pour aller à confesse. Mais une fois au confessionnal, je trouvai un bon prêtre qui confessait par routine. Lors, mon cœur se ferma et je ne pus me confesser selon les touches que j'avais eues. Je répondais seulement aux interrogatoires qu'il me faisait et écoutais ses remontrances mais de moi-même je ne lui pouvais rien dire. ${ }^{34}$

Rien dans ce qu'écrit Marie de l'Incarnation ne permet de savoir si les « récréations » qu'elle prenait avec ses compagnes avaient un caractère sexuel, n'était le rapprochement qu'on peut faire entre ces aveux et ceux de Jeanne Guyon et Marcelline Pauper. Mais le flou qui entoure ces souvenirs indique bien que les frontières assignées à "ce à quoi" on devait faire silence étaient tellement extensibles que celles-ci pouvaient aussi bien inclure des jeux totalement puérils que, de fait, nombre de fillettes pieuses fuyaient absolument, se réfugiant dès leur âge le plus tendre dans l'absolue solitude de leur chambre ou grenier ${ }^{35}$. Regarder quelqu'un dans les yeux, écouter des paroles gaies, se coudoyer, s'embrasser, n'était-ce pas déjà "pécher"?

\section{Savoir ou ignorer, mais feindre ignorer}

Dans les biographies religieuses, l'élément déclencheur de la conversion est souvent le choix que les familles font du mariage pour leur fille : conformément à une tradition qui remonte à l'hagiographie médiévale, la prise de conscience et l'épreuve de force avec les parents interviennent fréquemment au moment où il faut faire le deuil de la virginité. Cependant un petit nombre de récits concerne des femmes qui, avant de choisir d'entrer au couvent, ont été mariées ou bien ont eu une conduite exemplaire durant leur mariage et surtout leur veuvage. Les relations sexuelles au sein du mariage sont en général voilées de silence, que ce mariage ait été heureux comme ce fut le cas de celui de Marie de l'Incarnation, unie quelques mois seulement à un époux qu'elle dit avoir aimé beaucoup malgré sa «très grande aversion du mariage $\aleph^{36}$, ou qu'il ait été malheureux comme

\footnotetext{
34 Jamet $1932: 7-8$.

35 Conduites similaires chez les petites espagnoles décrites par Isabelle Poutrin $1987: 331-354$.

36 Jamet $1932: 11$.
} 
celui de mademoiselle de Neuvillars, unie à un huguenot qu'elle ne réussit pas à convertir ${ }^{37}$.

Seule de toutes celles-ci, Jeanne Marie Guyon, qui fut mariée à seize ans à un homme beaucoup plus âgé qu'elle, a laissé deviner ce que fut pour elle, dans les premiers temps de son mariage, la découverte des relations sexuelles conjugales. Son entrée dans les noces fut surtout marquée par un grand soulagement d'être unie à un homme : les relations conjugales n'allaient-elles pas la "guérir" de ses habitudes antérieures et apaiser « la crainte [qu'elle] avait de retomber dans les péchés»? Cependant, ce soulagement restait mêlé : «Quoique je fusse bien aise d'être mariée, je ne laissai pas de rester tout le temps que je fus accordée, de même longtemps après mon mariage, dans une extrême confusion. Elle venait de deux causes : la première était cette pudeur naturelle dont j'ai parlé que je ne perdis point pour mes péchés, parce que ces péchés étaient sans complices et que j'avais beaucoup de retenue avec les hommes $»^{38}$, écrit-elle. On imagine bien que la retenue corporelle inculquée aux filles dès le plus jeune âge, ce que le siècle appelle "la naturelle modestie des femmes", devait rendre banale cette appréhension quoique ce témoignage reste exceptionnel par sa franchise. Mais s'y mêlent d'autres peurs, crainte que les habitudes passées de la petite fille ne soient réitérées dans le sein du mariage, peur peut-être que le mari ne se doute de quelque chose, terreur surtout que l'absence d'aveu ne vienne entacher la pureté requise à l'entrée du mariage.

Pour Jeanne Marie Guyon, ces premiers temps de mariage sont un temps de prise de conscience du pouvoir sexuel qu'elle exerce sur son mari. Ce pouvoir, elle l'a déjà expérimenté - ce qui contribue aussi à cette confusion qu'elle ressent au moment de ses noces - avec un jeune homme auquel son père avait voulu la marier. Quoiqu'elle ait dédaigné cette proposition, ce jeune homme étant de grande qualité mais désargenté, elle avoue avoir joué de coquetterie avec lui :

Je couchais dans la chambre de mon frère, et entre la chambre de mon frère et la mienne, il y avait une porte condamnée qui donnait derrière mon lit, il se tenait toute la nuit à cette porte à me conter des

\footnotetext{
37 Du Sault 1649: 61.

38 Guyon 2001 : 161. Les italiques indiquent les passages censurés.
} 
extravagances, et quoiqu'il fût nu en chemise, et qu'il fît un froid très rigoureux, il ne s'apercevait pas du froid tant il était passionné. Je me divertissais à le laisser geler ; rien ne le pouvait arrêter, je fis des péchés sans nombre parce que souffrant de ses sottises et m'en divertissant je donnais occasion à des crimes effroyables que je savais qu'il commettait, mais je ne croyais pas qu'il y eût du mal d'être cause que les autres vous offensassent, ô mon Dieu, pourvu que je ne le fisse pas moi-même, comme si celui qui fait faire un mal n'est pas aussi coupable que celui qui le fait ; j'en commettais moi-même dans le secret, et je me contentais de n'avoir aucun complice de mes maux et qu'ils ne fussent sus de personne, et je ne croyais pas que vous me voyiez, ô mon Dieu, vous dont les yeux sont si purs. ${ }^{39}$

Ces ardeurs amoureuses, elle les retrouve chez son mari et s'en étonne d'autant qu'elle ne les partage pas : «Ce qui me paraissait plus étrange est que sitôt que mon mari était seul avec moi, il entrait dans une passion d'amour si violente pour moi que cela m'était même à charge. Il n'y avait rien alors que j'eusse pu exiger de lui qu'il ne m'eût accordé». Sa froideur s'accroît pourtant dans la mesure où elle constate que ces promesses durent peu. Son charme ne suffit pas à contrecarrer l'influence de sa belle-mère sur son époux qui reprend ses méchantes façons d'agir dès qu'il quitte ses bras. Depuis le début de leur union, les mauvais traitements qu'elle subit de la part de sa belle-mère joints au fait qu'elle ressent une sorte de mépris pour les manières qui règnent dans sa nouvelle famille l'ont plongée dans la tristesse. Dans les relations sexuelles, c'est la feinte, donc une nouvelle forme de silence, qui s'impose à elle :

J'avoue aussi que l'accablement de douleur où j'étais me rendait peu sensible à ses feux, quoique je ne le lui témoignasse pas et que je tâchasse de faire ce que je devais. Il est vrai aussi qu'étant encore si jeune, la douleur ne faisait pas une si profonde plaie que dans un âge plus avancé, que je me laissais gagner à ses caresses espérant qu'il m'aimerait mieux et qu'il n'aurait pas de dureté avec moi. ${ }^{40}$

Cette stratégie de la feinte, Madame Guyon l'adopta durant tout le temps qu'elle resta mariée pour tenter de garder la chasteté à laquelle elle aspirait. Elle constate que dès la deuxième année de son mariage, elle n'a plus été sensible au plaisir sexuel, ce qui s'est produit «avec

\footnotetext{
39 Ibid. : 158-159.

40 Ibid. : 167.
} 
un amortissement entier de la vivacité du sentiment pour toutes les fonctions [alimentaires et sexuelles]» et, ajoute-t-elle, «je crois que j'en dis assez pour me faire entendre $»^{41}$.

Ayant décidé de traverser le mariage en éprouvant le moins de plaisir possible, Madame Guyon continua de se montrer extrêmement circonspecte vis-à-vis des confesseurs trop rigoristes ou maladroits. Elle relate qu'au cours d'un voyage, elle se rendit chez un confesseur qu'elle ne connaissait pas et qui lui apprit qu'elle était en état de péché mortel car elle s'était mariée par devoir et non pour avoir des enfants. Par ailleurs, ajoute-t-elle, «il m'apprenait, sous prétexte de m’instruire, des péchés que j’avais ignorés jusqu'alors $»^{42}$. Réflexion qui rejoint certaines réserves des confesseurs eux-mêmes sur l'usage d'une confession qui pourrait se révéler trop instructive, réflexion qui explique peut-être pourquoi d'autres de ces confesseurs constatent que les femmes sont en général plus réticentes que les hommes à confesser leur "péché de chair" 43 . Comme beaucoup d'autres femmes dévotes mariées alors qu'elles eussent préféré entrer en religion, Madame Guyon chercha surtout à préserver son ignorance et à ne pas aller au-delà d'un certain savoir minimal sur les relations sexuelles. Tel était aussi le cas de Madame de Veuilly dont le biographe prétend que, devenue veuve et entrée en religion sous le nom de Marie de Saint Charles, elle « estoit si peu instruite aux combats et aux peines contraires à la chasteté ${ }^{44}$ qu'elle en était presque ignorante. Ici, il faut souligner que la transposition dans le sein du mariage de modèles conventuels de piété et de mortification qui visaient à la "mort des sens" devait contribuer à développer ce genre de conduite conjugale chez des femmes qui avaient généralement été élevées dans des couvents et regrettaient de n'y être pas restées définitivement.

Cependant certaines femmes recouraient au contraire à la confession ou du moins "se faisaient instruire" dans la mesure où elles craignaient d'être exposées dans le sein même de leur mariage au "péché". Cette démarche se trouve consignée dans les procès pour

\footnotetext{
41 Ibid. : 291.

42 Ibid. : 255.

43 Voir Delumeau 1990 : 22-23 ; Bernos 1992 : 413-426.

44 Léon de Saint Jean $1671: 317$.
} 
annulation de mariage qu'il nous faut évoquer désormais. Quoiqu'on ne sache rien de l'intensité de la piété religieuse des femmes qui s'adressaient aux tribunaux d'Église, il y a chez elles une semblable évocation de l'ignorance dont elles étaient sujettes à l'orée de leur mariage. Magdeleine Lhermitte était la fille de Jean Baptiste Lhermitte de Souliers, chevalier, seigneur de Vauzelle, gentilhomme servant du roi, et de damoiselle Marie Courtin de la Dehors et elle avait été mariée à treize ou quatorze ans, à Pierre Le Fuzelier, écuyer. En 1663, elle vint se plaindre devant l'Official de Saint-Germain-des-Prés que, depuis huit ans qu'elle était mariée, son mariage n'avait pas été consommé. Elle déclara que, durant les premiers temps de son mariage, elle «ne sçavoit dans son ignorance et le peu d'expérience qu'elle avoit alors, ce qu'estoit le mariage ». Elle s'était alors adressée à « des personnes de piété » qui lui donnèrent «quelques conférences» et lui firent comprendre que ce qui se passait entre elle et son mari ne la mettait pas en "seureté $»^{45}$. Plus inquiète encore pour le salut de son âme, Élisabeth Guyot était venue en 1617 exposer que ses relations avec son mari lui avaient «fait naistre un scrupulle en l'âme et en sa conscience qui l'oblige[ait] à s'en plaindre ». En effet, avant d'aller devant le juge d'Église, elle avait «apprins qu'elle commectoit un péché mortel que de consentir à de telles pollutions» et elle ajoutait qu'elle s'était mariée "pour estre mère et non pour adultère $»^{46}$, rappelant ainsi la différence canonique entre la sexualité maritale entièrement vouée à la procréation et celle qui avait cours hors du mariage, qui était dominée par la recherche du plaisir. C’est cependant non auprès d'un confesseur qu'elle chercha à s'instruire. Peut-être comme Jeanne Guyon répugnait-elle à cet acte. Peut-être avait-elle peur d'en apprendre trop. C'est donc auprès de sa mère et de ses sœurs déjà mariées que cette femme, fille d'un vendeur et contrôleur de vin, bourgeois de Paris, était allée "prendre advis et conseil», ce qui ne l'empêchait pas d'invoquer son «âme», sa «conscience» et le «péché mortel». Avis et conseils bien tardifs puisqu'elle s'était mariée trois ans auparavant à l'âge de vingt-deux ans, tandis que Magdeleine Lhermitte mariée à un âge à peine nubile,

\footnotetext{
45 AN, Z1O 243, dossier Lhermitte-Le Fuzelier, 23 mai 1663.

46 AN, Z1O 99, dossier Guyot-Fueillet, 21 juin 1617.
} 
était demeurée sans se plaindre huit ans avec son époux. Que ces femmes aient été ou non si ignorantes qu'elles le disent en entrant dans la vie conjugale importe moins que de constater qu'elles se devaient de garder le silence sur leur initiation et sur ce qui se passait dans l'intimité de leur couple durant de longues années. Bien entendu, il faudrait pouvoir faire la part des "stratégies" utilisées par les plaignantes pour rendre leur démarche valide devant l'Official. Pour qu'une épouse fût entendue, mieux valait qu'elle apparût tout à fait innocente et qu'elle pût affirmer qu'elle n'avait compris ce qui lui arrivait qu'au bout d'un certain temps - en principe fixé à trois ans dans la procédure -, sauf à passer pour une fille déjà affranchie. C'est du moins ce que la partie adverse, l'époux, pouvait faire valoir pour sa défense. On le voit dans un plaidoyer prononcé au début du XVII siècle, où le défenseur du mari essaie ainsi de piéger l'épouse de dixhuit ans avec les arguments suivants :

On pourrait volontiers demander où est-ce qu'elle a appris, en quoy la frigidité est différente d'un embrassement plein d'amour, qu'est-ce qui peut apporter contentement pour saouller l'appetit en traictant de l'amour (...) ou bien estant vierge elle ne sçait encore ce que c'est que l'amour, et se plaint d'une chose qu'elle n'a point expérimentée, ou bien si elle se monstre autre qu'elle n'est, sera-t-elle si impudente en plein jugement qu'elle, se sentant en sa conscience n'avoir point gardé sa virginité, ose sans crainte se prévaloir de l'espérance d'un mariage légitime ?47

De même le défenseur du mari d'une très jeune femme de quinze ans, Marie Bostel, estime-t-il que «en cette jeunesse les jeunes filles ou femmes sçavent peu que c'est des œuvres du mariage le temps leur en donnant plus grande cognoissance de sorte que se plaindre en cette aage il y a apparence qu'il y a de l'animosité et suscitation en cette poursuitte $»^{48}$.

Cependant, la question que posent ces opinions de juriste est moins de savoir si ces femmes étaient réellement si ignorantes qu'elles prétendaient l'être que de constater qu'elles devaient se montrer ignorantes. Quoique mariées toutes deux très jeunes, et même plus jeunes que la plupart des filles de cette époque, peut-être avaient-elles,

\footnotetext{
$47 \quad$ Robert 1611 : 556-557.

48 AN, Z1O 99, dossier Andrenas-Bostel, 6 février 1617.
} 
à l'instar de Jeanne Marie Guyon, tout de même flirté avec des jeunes gens cachés (ou non) derrière une porte ?49 Peut-être avaient-elles été affranchies par certaines de leurs compagnes comme le roman libertin le plus célèbre du XVII ${ }^{\mathrm{e}}$ siècle, L'École des filles, le suggère en mettant en scène une oie blanche instruite par l'une de ses malicieuses cousines ${ }^{50}$ Peu importe en vérité car, dans le sein du mariage, il leur fallait désormais faire comme si elles n'eussent jamais rien su des relations sexuelles. C'est d'ailleurs aussi l'un des messages de L'École des filles - dont le titre lui-même dit assez que le libertinage commence quand les filles se mettaient à s'instruire : apprends, dit en substance Suzanne à Fanchon, mais surtout, ne dis à personne que tu sais.

\section{Ce que le silence fait aux époux}

On aurait tort de penser que si Magdeleine Lhermitte et Élisabeth Guyot cherchaient à se renseigner sur la sexualité conjugale, c'était pour repousser les limites de la connaissance. En réalité, tout indique au contraire que les femmes qui venaient se plaindre devant l'Official ne se plaignaient pas seulement de ce que leur mari était impuissant mais aussi qu'il leur avait fait connaître des pratiques sexuelles qu'elles auraient préféré ignorer ${ }^{51}$. L'ignorance supposée des jeunes épousées avait pour effet, et même pour objet, de faire de l'homme l'éducateur de sa femme. C'était lui qui était censé maîtriser un certain savoir sur la sexualité. Dans cette mesure, il attendait de son épouse qu'elle ne se dérobât pas à lui et qu'elle lui obéît au cours de son initiation, et audelà. Aussi, devant le tribunal de l'Officialité, certains maris cherchent-ils à marquer des points en se plaignant de n'avoir pas été suffisamment obéis par leur femme qui exprimait de la répugnance vis-à-vis d'eux, ce qui a d'ailleurs une certaine vraisemblance étant donné que beaucoup d'époux s'étaient à peine vus avant d'entrer dans le mariage. Quant aux femmes, elles anticipent souvent cette accusation en mettant en avant qu'elles se sont toujours soumises aux

\footnotetext{
49 Travaux classiques de Jean-Louis Flandrin sur la fréquentation prénuptiale.

50 L'École des filles, édition Jacques Prévot 1998. Sur ce roman, voir Muchembled 2005 : $111-112$ et $139-143$.

51 Recommandations faites par les confesseurs notamment par François de Sales aux maris de ne pas trop en apprendre à leurs épouses. Voir Bernos 1985 : 162.
} 
désirs de leur époux, sans se rebeller. Ainsi Marie Bostel, cette jeune femme qui n'a que quinze ans et deux mois quand elle vient se plaindre devant l'Official en 1617, déclare-t-elle à plusieurs reprises qu'il n'a pas tenu à elle que son mari n'ait pas eu sa "compagnie » mais « qu'elle luy a rendu toutes les obéissances et celles qu'il a voullu et désiré et ne l'a empesché de rien à toutes les foys qu'il luy a requis $\aleph^{52}$.

C'est ce devoir d'obéissance ainsi que le silence auquel elles sont tenues qui leur fait admettre dans un premier temps tous les agissements de leurs maris ainsi que les quelques explications qu'ils veulent bien leur donner. Ce que décrivent les épouses qui viennent se plaindre devant le tribunal d'Église, ce sont donc des hommes qui, avant toute chose, exercent des droits sur leur corps. Dans certains cas, très rares cependant, l'épouse déclare ne pas avoir souffert véritablement de ce que les leçons de son maitre aient été marquées du sceau du "péché" parce qu'elle n’a pas été violentée. Magdeleine Lhermitte, dont on a vu qu'elle avait été mariée à treize ou quatorze ans, déclare ainsi que si les personnes de piété qu'elle avait rencontrées en venant s'installer à Paris ne l'avaient instruite, elle «seroit demeurée avec ledit Le Fuzelier beu mangé et couché avec luy, souffert ses caresses extérieures, et vescu en pareille intelligence que s'il eust esté son véritable mary ayant tousjours ignoré ce que estoit la consommation effective du mariage, et dans ceste ignorance n'en avoit jamais formé aucune plainte ». Elle ajoute encore que, ce que son mari « luy faisoit croire estre la consommation » n'avaient été «que des attouchements sans effects, tout ce qui estoit nécessaire pour cette consommation y ayant manqué $»^{53}$. Elle vécut dans ce silence durant huit ans, ce qui laisse à penser qu'elle pouvait trouver du contentement voire du plaisir à ces «caresses extérieures» et ces «attouchements sans effects», prodigués sans violence et apparemment au sein de cette douce intimité que les archives résument toujours par le fait d'avoir «bu, mangé et couché ensemble ». On notera que Magdeleine Lhermitte ne considérait pas ces caresses comme peccamineuses, n'était qu'elles n'aboutissaient

\footnotetext{
52 AN, Z1O 99, dossier Andrenas-Bostel, 6 février 1617.

53 AN, ZIO 243, dossier L'Hermitte-Le Fuzelier, 23 mai 1663.
} 
pas à la consommation entière du mariage. En ce sens, son sentiment était tout à fait conforme aux prescriptions des confesseurs qui acceptaient les caresses et la stimulation clitoridienne, qui, pensaientils, pouvaient favoriser l'émission de semence féminine, mais à l'expresse condition qu'elles fussent suivies d'un coït complet ${ }^{54}$. Mais la situation de Magdeleine Lhermitte, plus généralement, indique aussi que les couples qui s'entendaient bien pouvaient profiter du silence qui entourait leurs relations sexuelles pour se livrer à des caresses ou des embrassements que l'Église n'approuvait pas forcément.

Plus nombreuses sont les femmes qui décrivent la manière dont elles ont été forcées de subir toutes sortes d'attouchements brutaux, non pas destinés à susciter du plaisir, mais à conquérir à toute force leur virginité. Claude Manzangerbe, une servante de vingt-huit ans, mariée depuis neuf ans, se plaint devant l'Official de Paris que, si le rapport médical la trouve "corrompue », c'est que son mari, Claude Bonamy, «luy auroit fait mil indignités luy mettant les doigts dedans sa nature la forçant et égratignant par dedans » et elle se plaint d'avoir été longuement soignée par un médecin "pource qu'il la travailloit tellement par le ventre qu'il luy en faisoit perdre presque la veue oultre les cuissons qu'elle a (souffert) dedans sa nature par les effortz qu'il luy a faitz tant de ses doigts que de son corps $»^{55}$. Jehanne Chevauchée, mariée depuis deux ans et demi, déclare que son mari a « tascher de la corrompre avec ses doigts et avec ses mains, mesme est vray de dire que au lict il l'a violentée avec des chandelles et des cirges qu'il luy mettait dans la partye genitalle et la mordoit au lict, la chassant hors du lict, la tiroit en bas sur le planchet, la faisoit metre nue dans tonneaux la nuit en la boutique $»^{56}$. Élisabeth Guyot dit que son mari Fueillet, lui expliquait «qu'elle estoit trop estroitte ». Elle précise que, comme il lui «mettoit le doigt pour dilater et ouvryr», en vertu de son obéissance, « elle luy a quelque foys permis mays qu'elle ne pouvoit plus endurer le mal qu'il luy faisoit et voullant remettre le doigt elle luy a reffusé et à cause de ce qu'il l'a battue et excedder par

54 Voir Flandrin 1981 : 133-135. Sur la théorie médicale de la double semence et du double plaisir, voir McLaren 1985 : 323-341.

55 AN, Z1O 98, dossier Manzangerbe-Bonamy, 6 juillet 1616.

56 AN, Z1O 98, dossier Chevauchée-Lefebure, 29 août 1616. 
plusieurs foys jusqu'à luy démettre le petit doigt $\aleph^{57}$. La défloration est évidemment un enjeu de taille dans ces récits pour toutes sortes de raison. Si la femme qui est examinée par les experts médicaux, médecins, chirurgiens et sages-femmes qui exercent auprès du tribunal, est déclarée "corrompue»-terme qui indique assez la porosité entre la vision cléricale et l'appréhension médicale de la sexualité - alors qu'elle a entamé une procédure pour prouver l'impuissance de son mari, il lui faut insister au préalable sur le fait que cette défloration est le fruit d'attouchements violents. Mais il est difficile de croire que l'insistance que mettent les plaignantes à décrire de tels agissements ne répond qu'à une stratégie procédurière. Ce n'est pas seulement la consommation du mariage qui est un défi pour ces hommes nouvellement mariés, par ailleurs désemparés et souvent rendus enragés par leur propre impuissance, mais aussi le ravissement de l'hymen. Quoique certains médecins depuis le XVIe siècle au moins aient douté de l'existence de cette membrane, non seulement ceux qui examinaient les femmes pour l'Officialité n'en doutaient pas ${ }^{58}$ puisqu'ils étaient capables de constater qu'une fille était «entière, vierge et non corrompue ", suivant l'expression consacrée, mais ils considéraient qu'il fallait au membre viril une force spéciale pour déflorer une fille « entière ». Il n'y a pas de raison de penser que les maris à l'entrée du mariage ne concevaient pas eux-mêmes leur devoir de cette manière-là : l'un d'eux supplie la cour de lui permettre de se remarier avec une femme veuve, moins difficile à pénétrer ${ }^{59}$. En tant que telle la perte de la virginité devait être le triomphe du mari, la «corruption» de son épouse signifiant que son corps lui appartenait désormais et la perte de son "entièreté" étant la marque de sa virilité. N'est-ce pas la véritable signification de ces déflorations manuelles et de certains sévices destinés à faire couler le sang et à mimer la pénétration, comme le fait de piquer le corps de la femme avec des épingles ?60

\footnotetext{
57 AN, Z1O 99, dossier Guyot-Fueillet, 21 juin 1617.

58 Darmon 1979: 164-181.

59 AN, Z1O 100, dossier Andrenas-Bostel, 21 juillet 1617.

60 AN, Z1O 99, dossier Andrenas-Bostel, 6 février 1617.
} 
Si les femmes rompaient le silence pour exposer très précisément les violences qu'elles avaient endurées dans leur corps, elles avaient davantage de mal à avouer d'autres activités sexuelles dont elles avaient été les témoins. Nicole Barbier, vingt-cinq ans, fille d'un cordonnier, franchit un petit pas en déclarant au juge qu'«elle a souffert [de son mari] une infinité de tourmentz (...) oultre les injures et excès qu'il luy a faitz et les salles actions qu'il commet devant elle qui luy font mal au cœur et que par honnesteté elle n'auze dire ${ }^{61}$. Claude Manzangerbe « dict qu'il [son mari] luy a dict que estant jeune et auparavant que de se maryer il s'est corrompu et gaster de luy mesme et qu'il luy a connus tant de salletés et de déshonnêtetés qu'elle est contrainte par honnesteté de se tayre tant elles sont vilaines et honteuses. » Il leur faut cependant être précises si elles veulent que leur plainte aboutisse à une annulation de mariage. Aussi plusieurs épouses finissent-elles par décrire des comportements là encore récurrents, résumés dans le témoignage de Claude Manzangerbe :

a dict que depuis qu'ils sont mariez toutes les foys qu'il a voullu jouyr d'elle charnellement il s'est tousjours excité de soy mesme tirant sa nature en sa main pour la mettre en estat estant perpetuellement à se bransler dedans le lict et que aussytot qu'il ostoit sa main sa nature s'affaiblissoyt tellement que par après il estoyt une grande heure autour d'elle à la travailler et molester toute la nuit une infinité de reprinses sur elle par les mesmes voyes excitementz et branlementz. ${ }^{62}$

Là encore, il faut faire la part de la "stratégie" des plaignantes. Une telle description ne peut qu'indiquer au juge qu'elles sont en danger si elles continuent à cohabiter avec des maris suffisamment débauchés pour commettre le "péché d'Onan" dans le lit conjugal (ou plus exactement tenter de le commettre car le "péché" consiste surtout à émettre la semence hors du "vaisseau » ou «vase » idoine) mais aussi pour donner à voir à leur compagne un spectacle qu'elles n'ont pas à contempler. L'une d'elle, Marie Bostel précise toutefois qu'elle s'est rendu compte de l'état de son mari du fait que " par plusieurs foioys il la luy a baillée à manier ce qu'elle a fait ». D'après les juges d'Église, les épouses ne sont pas censées toucher le sexe de leur mari-et on

\footnotetext{
61 AN, Z1O 98, dossier Barbier-Vignardet, 9 juillet 1616.

62 AN, Z1O 98, dossier Manzangerbe-Bonamy, 6 juillet 1616.
} 
remarquera que si Marie Bostel l'a fait, c'est à sa demande expresse et pour lui obéir-, sans enfreindre les règles de la pudeur conjugale. Dans une affaire d'impuissance où il se trouve que le mari n'a qu'un testicule dans le scrotum, le juriste Sébastien Roulliard blâme la femme de l'avoir découvert car :

La loy susdite [le Deutéronome] défend à la femme de ne jetter les yeux, ou les mains pétulantes aux parties, où la honte de son mary se cache (...) N'en fut-il d'autre exemple, que celuy lequel Pline en son Epistre 24 du 6 livre fait sonner si haut, d'une chaste matrone du bourg de Larie, qui ressentant mille angoisse en son âme, des pénibles tourmens que souffroit son mary par un ulcère qui luy pourissoit les parties génitales : ne peut néantmoins jamais se résouldre à prendre la hardiesse de voir ce que c'estoit, sinon à toute extrémité, pour discouvrir s'il y avoit quelque espérance de guérison. ${ }^{63}$

L'aveu de Marie Bostel n'est donc pas particulièrement habile, sauf à considérer qu'elle met en avant son obéissance aveugle pour apparaitre comme le jouet d'un homme dangereux. Mais au-delà des visées stratégiques de ces aveux, leur concordance sur le point de qualifier de «saletés » les efforts manuels des époux pour parvenir à l'érection et au coït indique bien que, si les femmes ont intériorisé leur nécessaire obéissance à leurs maris, elles disent attendre d'eux qu'ils exercent leur pouvoir sur leur corps sans les amener ellesmêmes à "pécher" ni par la vue, ni par le toucher. Lorsqu'ils les poussent à agir ainsi, elles soulignent qu'elles n'en ont pas pris l'initiative: ce sont toujours elles qui «souffrent» leurs caresses. Passives, c'est ainsi qu'elles se présentent, et sans doute est-ce ainsi qu'elles ressentent leur rôle dans le "combat vénérien", même si la pratique peut être autre. Ici, on ne peut que constater la convergence absolue entre les discours de l'Église sur la sexualité et le ressenti que les femmes expriment. Convergence logique dans la mesure où la parole se dit dans un cadre religieux, à la demande d'un directeur de conscience et devant un juge d'Église mais convergence que l'on peut aussi attribuer au fait qu'à tout prendre - et à supposer qu'on puisse endosser un discours par pure stratégie - se présenter comme passive dans une affaire d'impuissance était sans doute plus valorisant que de venir se plaindre de ce que sa «matrice assoiffée » réclamait son dû,

63 Roulliard $1610: 241 \mathrm{r}^{\circ}$. 
comme tout une série de textes plus ou moins pamphlétaires tendaient à l'expliquer, à propos des cas judiciaires d'impuissance ${ }^{64}$.

Sans doute les bribes de paroles de femme recueillies grâce aux biographies spirituelles et aux procès de justice confirment-elles, chacune dans ses formes propres d'énonciation, que le silence a été un puissant instrument de contrôle de l'Église sur la sexualité, conformément à l'analyse qu'en faisait Michel Foucault dans La $V$ olonté de savoir. Elles montrent aussi qu'il fut un vecteur du pouvoir des hommes sur le corps de leurs épouses, suivant un partage sexué du savoir sur la sexualité et du pouvoir de s'exprimer sur cette question. Encore faudrait-il avoir une attention plus aiguë aux savoirs des hommes et à leur propre silence. Dans l'interaction entre discours et pratiques, le silence joue lui aussi son rôle : quand le silence se fait, le recours à des fantasmes qui superposent le langage symbolique et les réalités du corps, la crainte de la transgression et de la faute, la passivité accompagnent l'intériorisation des interdits et leur inscription corporelle dans les attitudes et les comportements les plus intimes. Il est cependant douteux que ce type de processus soit né au XVII ${ }^{e}$ siècle, ce que nous savons de la sexualité au Moyen Âge ${ }^{65}$ n'incitant pas à penser qu'il s'agisse d'une véritable innovation du Grand siècle, si ce n'est le raffinement qui est mis à exprimer une telle intériorisation. Un tel vécu était-il partagé par toutes les femmes? Force est de constater que nous ne saurions dire grand chose de ce que ressentaient la bouchère blagueuse ou cette petite fille qui aimait tant faire rire les assemblées avec des mots à double sens, ou toutes celles qui apparaissent de loin en loin dans les pages des biographies religieuses ou les archives judiciaires : filles qui ont choisi de s'enfuir avec un séducteur, jeunes filles de famille enfermées aux Madeleines pour faire pénitence, prostituées qui suivent les armées en campagne, mondaines et coquettes en décolleté outré. Celles-là, dont on dit qu'elles parlaient et riaient du sexe, ont-elles quelque part laissé leurs mots?

64 Sur ce type de discours McLaren 2007 : 61-69.

65 Voir Karras 2005. 


\section{Sources imprimées citées}

Bourdin (le P. Matthieu), 1679, Vie et conduite spirituelle de la demoiselle Madeleine Vigneron seur du Tiers-Ordre de S. François de Paule suivant les Mémoires qu'elle en a laissez par ordre de son directeur, le tout recueilli par l'ordre de son directeur, Rouen, Bonaventure Le Brun.

Bouix (le P. Marcel), 1871, Vie de Marcelline Pauper de la congrégation des scurs de la Charité de Nevers écrite par elle-même, Nevers, Fay.

Du Sault (le P. Nicolas), 1649, La Vie de Mademoiselle de Neuvillars, miroir de perfection pour les femmes mariées et pour les âmes dévotes, Paris, Sébastien Cramoisy.

L'École des filles, 1998, édition Jacques Prévot, Libertins du XVIIe siècle, tome I, Paris, Gallimard.

L'Expérience mystique de Marcelline Pauper, religieuse de la congrégation des scurs de la Charité et de l'instruction chrétienne de Nevers, Étude critique de ses écrits. Avec le concours du père André Ravier s j., 1982, Nevers, Couvent saint Gildard.

Guyon Jeanne Marie, 2001, La Vie par elle-même et autres écrits biographiques, édition critique avec introduction et notes par Dominique Tronc, Paris, Honoré Champion.

Jamet (Dom Albert) (éd.), 1932, Le Témoignage de Marie de l'Incarnation, Ursuline de Tours et de Québec, Paris, Gabriel Beauchesne.

Léon, Fr. de Saint Jean, 1671, La Vie de la vénérable mère Marie de S. Charles religieuse de sainte Élizabeth, dite au siècle Madame la baronne de Veuilly, Paris, F. Muguet.

Piny Alexandre (R.P.), 1679, La Vie de la vénérable Mère Marie Magdeleine de la Très-sainte Trinité, fondatrice de l'odre de Notre-Dame de Miséricorde, Annecy, Jacques Clerc.

Robert Anne, 1611, Quatre livres des arrests et choses jugées par la court, Paris, J. Cottereau.

Roulliard Sébastien, 1610 (2e édition), Les Reliefs forenses, , Paris, Thomas de la Ruelle.

Thomas d'Aquin de Saint Joseph, religieux carme déchaussé, 1633, Histoire de la Vie et des vertus de la vénérable mère Galiote de Saint Anne de la très-illustre maison des comtes de $V$ aillac, religieuse de l'ordre de S. Jean de Hierusalem, prieure du monastère de l'Hospital de Beaulieu en Quercy, réformatrice de son ordre en France, Paris, Sébastien Hure.

Vie de damoizelle Elizabeth Ranquet, 1655, Paris, Charles Sacreux.

La Vie de la Mère Magdeleine de Saint Joseph, religieuse carmélite déchaussée de la première règle selon la réforme de Sainte Thérèse, 1670, nouvelle édition revue et augmentée, Paris, Pierre Le Petit. 
La Vie de la Vénérable Mère Agnez de Jésus religieuse de l'ordre de S. Dominique an dévot monastère de Sainte Catherine de Langeac par un prêtre du clergé, 1666, Paris, Georges Josse.

La Vie de Marguerite du Saint Sacrement, religieuse carmélite du monastère de Beaune, 1655, Paris, Pierre le Petit.

\section{Ouvrages cités}

ALBERT Jean-Pierre, 1997, Le sang et le ciel. Les saintes mystiques dans le monde cbrétien, Paris, Aubier.

Bernos Marcel, 1985, «Le concile de Trente et le temps des mises en ordre », in Marcel Bernos, Jean GuYON, Charles DE LA RonCIÈre \& Philippe LÉCRIVAIN (éd.), Le "Fruit défendu". Les Chrétiens et la sexualité de l'Antiquité à nos jours, Paris, Le Centurion, p. 143-203.

—, 1992, «La sexualité et les confesseurs à l'époque moderne », Revue de l'bistoire des religions, CCIX, 4, p. 413-426.

BROWN Peter, 1995, Le renoncement à la chair. Virginité, célibat et continence dans le christianisme primitif, traduit par Pierre-Emmanuel Dauzat et Christian Jacob, Paris, Gallimard.

BYNum Caroline, 1994, Jeûnes et festins sacrés. Les femmes et la nourriture dans la spiritualité médiévale, traduit par Claire Forestier Pergnier et Éliane Utudjian Saint-André, Paris, Le Cerf.

D’Avack Pietro A., 1949, « La copula perfecta e la consummatio coniugii nelle fonti e nella dottrina canonista classica », Rivista italiana per le scienze giuridiche, Milano, serie III, anno III, p. 163-250.

DARMON Pierre, 1979, Le tribunal de l'impuissance. Virilité et défaillances conjugales dans l'Ancienne France, Paris, Seuil.

DelumeAu Jean, 1990, L'aveu et le pardon. Les difficultés de la confession. XIII'-XVIII' siècle, Paris, Fayard.

Demars-Sion Véronique, 1986, À la recherche d'un père... Action en recherche de paternité et action de la femme séduite dans l'Ancien Droit français à travers la pratique du Nord, thèse de droit privé, Lille II.

DuCHÊNE Roger, 2004, Être femme an temps de Lonis XIV, Paris, Perrin.

FLANDRIN Jean-Louis, 1975, Les amours paysannes (XVIT-XIXe siècle), Paris, Gallimard/Julliard, collection Archives.

—, 1981, Le sexe et l'Occident. Évolution des attitudes et des comportements, Paris, Seuil. 
GÉLIS Jacques, 2005, «Le corps, l'Église et le sacré », in Alain CORBIN, Jean-Jacques Courtine \& Georges Vigarello (dir.), Histoire du corps, tome 1, De la Renaissance aux Lumières, Paris, Seuil, p. 17-107.

KARRAS Ruth Mazo, 2005, Sexuality in medieval Europe: doing unto others, New York/London, Routledge.

LE BRUN Jacques, 1986, «À corps perdu. Les biographies spirituelles féminines du XVII e siècle ", in Charles Malamoud \& Jean-Pierre Vernant (éd.), Corps des Dieux, Paris, Gallimard, p. 389-408.

MCLAREN Angus, 1985, "The pleasures of procreation: traditional and biomedical theories of conception ", in W.F. BYNUM \& Roy PORTER (eds), William Hunter and the eighteenth-century medical world, Cambridge, Cambridge University Press, p. 323341.

—, 2007, Impotence. A cultural History, Chicago/London, University of Chicago Press.

MillinAire Laurine, 2006, Les procès pour promesses de mariage. Enquête dans les Officialités de Rouen, de Fécamp et de Montivilliers (1569-1700), Master de l'Université de Rouen.

MuchembleD Robert, 2005, L'orgasme et l'Occident. Une histoire du plaisir du XV Te siècle à nos jours, Paris, Seuil.

Pellegrin Nicole, 2003, "L'écriture des stigmates (XVI $-\mathrm{XVIII}{ }^{\mathrm{e}}$ siècles) », in Pierre CORDiER \& Sébastien JAHAN (éd.), La Blessure corporelle. Violences et souffrances. Symboles et représentations, Les Cabiers du Gerbico, 4, Poitiers, p. 41-62.

POUTRIN Isabelle, 1987, «Souvenirs d'enfance. L'apprentissage de la sainteté dans l'Espagne moderne », Mélanges de la Casa de Velasquez, tome XXIII, p. 331-354. 